\title{
Electricity Cost Prediction using Autoregressive Integrated Moving Average (ARIMA) in Korea
}

\author{
Safdar $\mathrm{Ali}^{1}$ \\ Department of Software Engineering \\ University of Lahore, Lahore \\ Lahore, Pakistan
}

\author{
Do-Hyeun $\mathrm{Kim}^{2}$ \\ Department of Computer Engineering \\ Jeju National University \\ Jeju, Republic of Korea
}

\begin{abstract}
Electricity cost plays a vital role due to the immense increase in power utilization, rise in energy rates and alarms about the variations and impact on the environment which ultimately affects electricity cost. We claim that electrical power utilization data became more beneficial if it is presented to the customers along with the prediction of power consumption, prediction of energy prices and prediction of its expected electricity cost. It will assist the residents to alter their power utilization behavior, and thus will have an optimistic influence on the electricity production companies, dissemination network and electricity grid. In this study, we present a residential area power cost prediction by applying the Autoregressive Integrated Moving Average (ARIMA) technique in Korean apartments. We have investigated the energy utilization data on the foundation of daily, weekly and monthly power utilization. The accumulated data constructed on daily, weekly and monthly utilization are selected. Then we predict the maximum and average power consumption cost for each of the predicted daily, weekly and monthly power consumption. The power consumption and general price (General Electricity Price in Korea) data of Korea are used to analyze the efficiency of the prediction algorithm. The accuracy of the power cost prediction using the ARIMA model is verified using the absolute error.
\end{abstract}

Keywords-Electricity price; electricity cost; Autoregressive Integrated Moving Average (ARIMA); prediction; energy consumption

\section{INTRODUCTION}

Power cost prediction and forecasting have become one of the main areas of interest to the researchers and experts in energy markets due to the fluctuation of electricity cost. This leads to the requirement of accurate and efficient electricity cost prediction methodology. All the stakeholders of energy including, energy market players and electricity price regulators now think to give attention to the cost prediction and its evolution process. Similar to price prediction, market electricity cost estimation is vital information to organize bidding policies and increased their profits in electricity the market. Furthermore, it can also support customers to reduce their electricity expenses by appropriate consumption of power and the smart grid will be operated smoothly and efficiently with the satisfactory level of consumers' needs and power generation companies.

In the literature there are many approaches to price prediction that can be extended to the cost prediction. These techniques can be generally categorized into two classes. The

Funded by National Research Foundation (NRF) of Korea. Any correspondence associated to this paper should be directed to Dohyeun Kim first one is Artificial Neural Networks (ANNs) techniques. The second kind of price forecasting method is the time series approaches. ANN's techniques that possess outstanding strength and error clemency are the finest way to address the multi-layered nonlinear problems. ANN has acknowledged the responsiveness of investigators due to its rich modeling process, ease of implementation and decent performance in resolving linear and nonlinear problems. Until now it is effective to formulize and predicts variations in complicated power system using ANN techniques. Varies ANN-based techniques have been presented to estimate energy prices in almost all energy markets [1-6].

To intensify the forecasting correctness, it has been employed based on supervised neural learning methodologies $[7,8]$. These studies repeatedly applied neural network approaches, which comprises several attributes. These attributes are uninterruptedly examined by knowledge, and the approaches become hard to be established properly [9]. Moreover, it has been apparent that while the ANN models give minor inaccuracy throughout training the input data patterns, the inaccuracy in testing these patterns is typical of a greater order [10], in other ways we can say that this technique when applied to systems in the real world, the prediction accuracy is compromised to an unacceptable level. Moreover, this method is required to convert the characters of all the glitches into quantities and alter all the implications into the arithmetic calculation. Yet, it will certainly result in the loss of important data which will degrade the prediction accuracy.

The non-stationary time series approaches like Autoregressive Integrated Moving Average (ARIMA) [11], stationary time series models like autoregressive (AR) [12], Dynamic Regression (DR) and Transfer Function (TF) [13] are formulated previously for prediction of energy price. Stationary time series methods can be extended for forecasting of power costs. In the modest electricity power marketplaces, the series of electricity costs describes features like: great occurrence, non-constant average and adjustment, every day, weekly, monthly and seasonable schedule outcome on holidays and community vacations; great instability and a great proportion of infrequent power consumption cost [14]. It is not simple to forecast electricity costs precisely, consequently, it requires exceptional dealing in case of predicting electricity cost changes. A hybrid technique to estimate day-ahead electricity price is presented in [15]. The technique is constructed on wavelet transform, ARIMA method and Radial Basis Function Neural Networks (RBFN). 
The price estimating model for electric energy market stakeholders to minimize the danger of price volatility is proposed in [16]. The method integrates the Enhanced Probability Neural Network (EPNN), Probability Neural Network (PNN) and Orthogonal Experimental Design (OED). Another work described the fusion of feature selection method which is constructed on common information technique and wavelet transformation [17].

Some evolutionary algorithms are used for estimation in different fields of study which can also be used for electricity price and cost prediction. For example, a technique called sunflower optimization algorithm is used for prediction of circuit-based model known as proton exchange membrane fuel cell (PEMFC) [18]. The model is used to reduce the error of sum of squared of predicted and real output voltage. Another method proposed for the reduction of sum of the squared error for PEMFC is presented in [19]. Both models achieved acceptable results and minimized the gap between actual parameters and predicted parameters. A cost optimization model for hybrid energy system is presented in [20]. The model achieved better results as compared to existing methodologies. A multi-objective optimization algorithm for heat pump problem is proposed in [21]. The model produces better results.

In this paper we proposed electricity cost prediction methodology using ARIMA model in republic of Korea. The power consumption in Korean apartments and general electricity price are considered to evaluate the cost and its prediction accuracy. The prediction results of ARIMA $(0,1,1)$ model are evaluated using the absolute error (AE).

The rest of the paper is structured as follows. Section II describes the power consumption and cost prediction model. Section III explains the ARIMA-base cost prediction in detail. Section IV describes the case study of price prediction in Korea. Section V puts light on the analysis and result discussion and Section VI concludes the paper.

\section{POWER CONSUMPTION SCENARIO AND COST PREDICTION MODEL}

The power consumption scenario which is adopted for cost prediction is based on the different electricity power consumption of the day, week and month for the apartments of Hwasung building number 417 . We considered hourly power consumption data for the daily electricity cost prediction. For weekly basis cost prediction we considered power consumption on each day of the week and divided each day in three slots each of 8 hours. Then we calculated the maximum and average power consumption for each slot of the day. For monthly basis cost prediction we considered daily basis power consumption and calculated the maximum and average power consumption for each day of the month. The flow chart of the power consumption scenarios and power prediction model is shown in Fig. 1. The flow chart consists of several components. Smart meters are used to record the power consumption data of the building. The collected consumed power and price statistics are stored in database for further processing. The energy consumption and price data are loaded to the program for power cost prediction. After reading the data in the program, first the data is analyzed with respect to the duplication and if there is any duplicated data found we simply remove it by averaging technique. For each of the daily, weekly and monthly basis cost prediction we did not merely considered power consumption for one specific day, or week but we considered all the data of 30 days power consumption for each of the daily and weekly basis cost prediction.

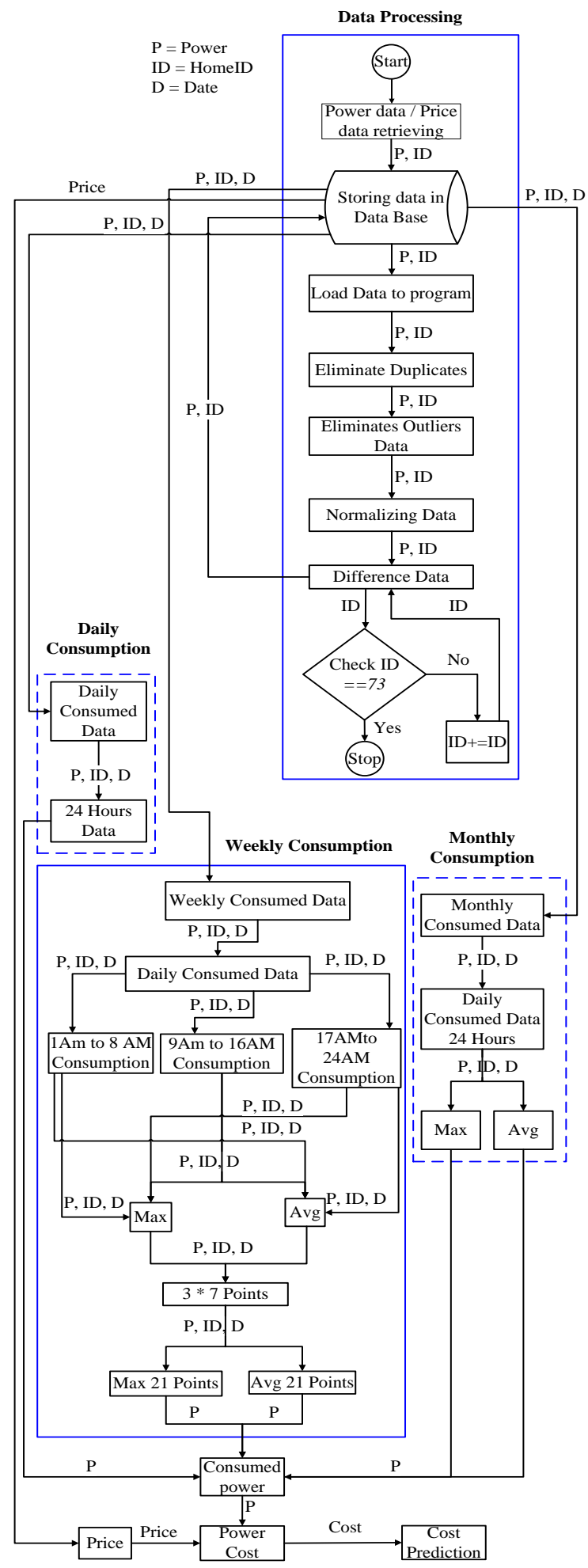

Fig. 1. Power Consumption Scenarios and Cost Prediction Model. 
For example, for daily basis power cost prediction we choose a representative daily basis power consumption patterns for the month of January 2010. The representative day is the average power consumption for that particular month against each hour of the whole month for all of the Hwaung building number 417 . Each corresponding hour of the day is considered and then we took the average of that hour for whole month. At the end we get a representative daily basis power consumption pattern for the month of January 2010.

Similarly, for weekly basis power consumption we considered daily basis power consumptions for all of the 30 days. The month is divided in to 4 weeks appropriately. Then we got one representative week for January 2010 by taking the average of each corresponding day of the week. At the end each day of the representative week is divided in to three slots as described in the start of this section. For monthly basis power cost prediction, we considered January 2010 data and then find the maximum and average power consumption during each day of the month.

\section{Cost PREDICTION USING ARIMA}

ARIMA $(0,1,1)$ forecasting method is one of the modified version of the ARIMA (p, d, q) model. The ARIMA (p, d, q) prediction method is the mutual category of modeling for predicting time series data. The model can be made static by using some kind of alterations like differencing and logging. In reality, one of the coolest method to talk regarding ARIMA technique is as fine-tuned kinds of random-walk and randomtrend technique. The fine-tuning contains adding lags of the differenced series and lags of the predicted errors to the forecasting equation as mandatory to remove any preceding touches of autocorrelation from the predicted errors. In ARIMA (p, d, q) model, $p$ is the number of autoregressive terms, $d$ is the number of non-seasonal variances, and $q$ is the number of lagged predicted error in the forecasting equation.

$\mathrm{Z}_{(\mathrm{k})}=\mu+\mathrm{Z}_{(\mathrm{t}-1)}-\beta \times \mathrm{e}_{(\mathrm{t}-1)}$

$\mu=\mathrm{Z}_{\mathrm{t}}-\mathrm{Z}_{(\mathrm{t}-1)}$

$\beta=1-\epsilon$

Where $Z_{(t)}$ is the estimation, $\beta$ is the coefficient of the lagged estimation error, $e_{(t-1)}$ represents the error at time epoch $t-1, \epsilon$ rate varies within limit $[0,1]$. To get optimal predicted values, we ran ARIMA $(0,1,1)$ technique twenty times for each 24-houres of the day and then took the average of the twenty points.

\section{A CASE STUdy In REPUBlic OF KoREA}

The proposed ARIMA $(0,1,1)$ based prediction is verified using a case study of predicting electricity cost on one day, one week and on monthly basis. The electricity price and power consumption data are collected on an hourly basis for a one month of January 2010 and then took an average of each corresponding hour to get one sample day of 24 hours. The cost for each hour is calculated. The unit of price is in Korean WON. Fig. 2 and 3 shows the 24 points of each hour for one day price and energy consumption, respectively. In this work, as an example we have shown only electricity price and power consumption data values of one day to calculate and then predict hourly basis one day power cost. In order to perform sufficiently and to show the flexibility and reliability of the ARIMA $(0,1,1)$ model for electricity cost prediction, section $\mathrm{V}$ shows that the ARIMA algorithm perform well when we consider maximum and average power consumption during each slot of the day on different week days. The hourly electricity cost values have been estimated based on the ARIMA $(0,1,1)$ model.

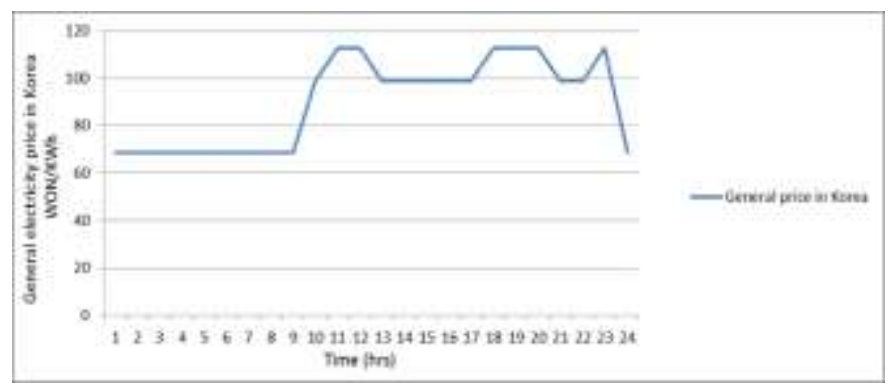

Fig. 2. General Electricity (Actual/Original) Prices, on (01/01/2010).

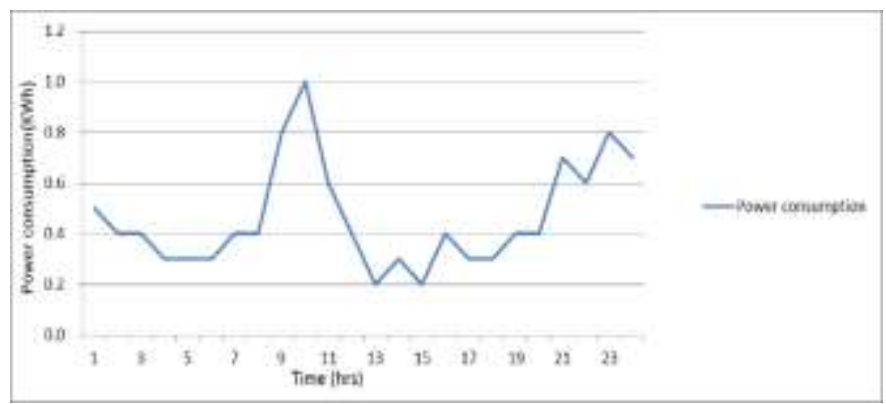

Fig. 3. Power/Electricity Consumption on (01/01/2010).

\section{ANALYSIS AND RESULt DISCUSSION}

In this section we are evaluating $\operatorname{ARIMA}(0,1,1)$ model performance with respect to electricity cost prediction. In the literature many objective functions exist to assess the prediction algorithm. We used absolute error (AE) method as the evaluation criteria. Like other objective functions, estimation performance of $\mathrm{AE}$ is much improved when the objective function value is minor. Equation (4) below describes the (AE) objective function.

$\mathrm{AE}=\left|\mathrm{p}_{\mathrm{p}}-\mathrm{a}_{\mathrm{p}}\right|$

In equation (4) $a_{p}$ means real time original/actual electricity cost, $p_{p}$ means predicted electricity cost.

Fig. 4 shows the daily basis actual cost and predicted cost based on the ARIMA $(0,1,1)$ technique for Hwasung building number 417 . Each hour of the day represents the average power cost of all homes of building number 417 . We have considered the experimental investigation of the electricity cost data by applying ARIMA $(0,1,1)$ method. In Fig. 4 there are three lines. Sky color line described electricity cost based on the general price in Korea during one representative day of January 2010, Indian red color shows the cost prediction line using ARIMA $(0,1,1)$ technique and yellow green color represents the absolute error for $\operatorname{ARIMA}(0,1,1)$ technique. From the Fig. 4 we can see that the prediction based on the ARIMA $(0,1,1)$ almost follows the actual cost line. If we see 
the absolute error line, then we can say that the absolute error line some time approaching to zero during some hours of the day, which means that the prediction of cost using proposed ARIMA $(0,1,1)$ method in this case up-to somehow follows the actual electricity cost line. As the prediction accuracy between actual and predicted cost increase, the absolute error decreases.

Fig. 5 shows the efficiency of the proposed ARIMA $(0,1$, 1) model based on the weekly and monthly power consumption scenario as shown in Fig. 1.

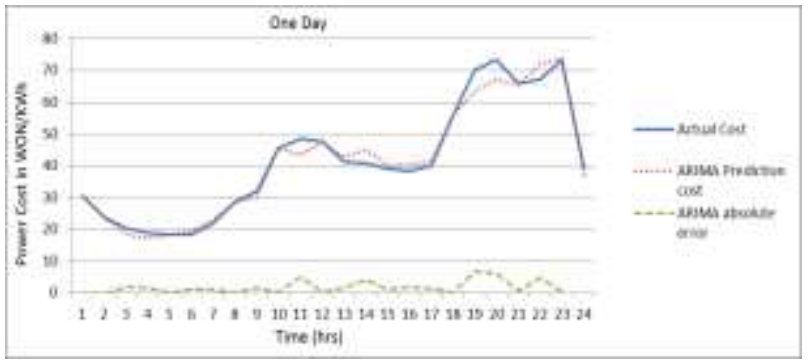

Fig. 4. Actual Cost, Predicted Cost using ARIMA $(0,1,1)$ Model and Absolute Error for One Day.

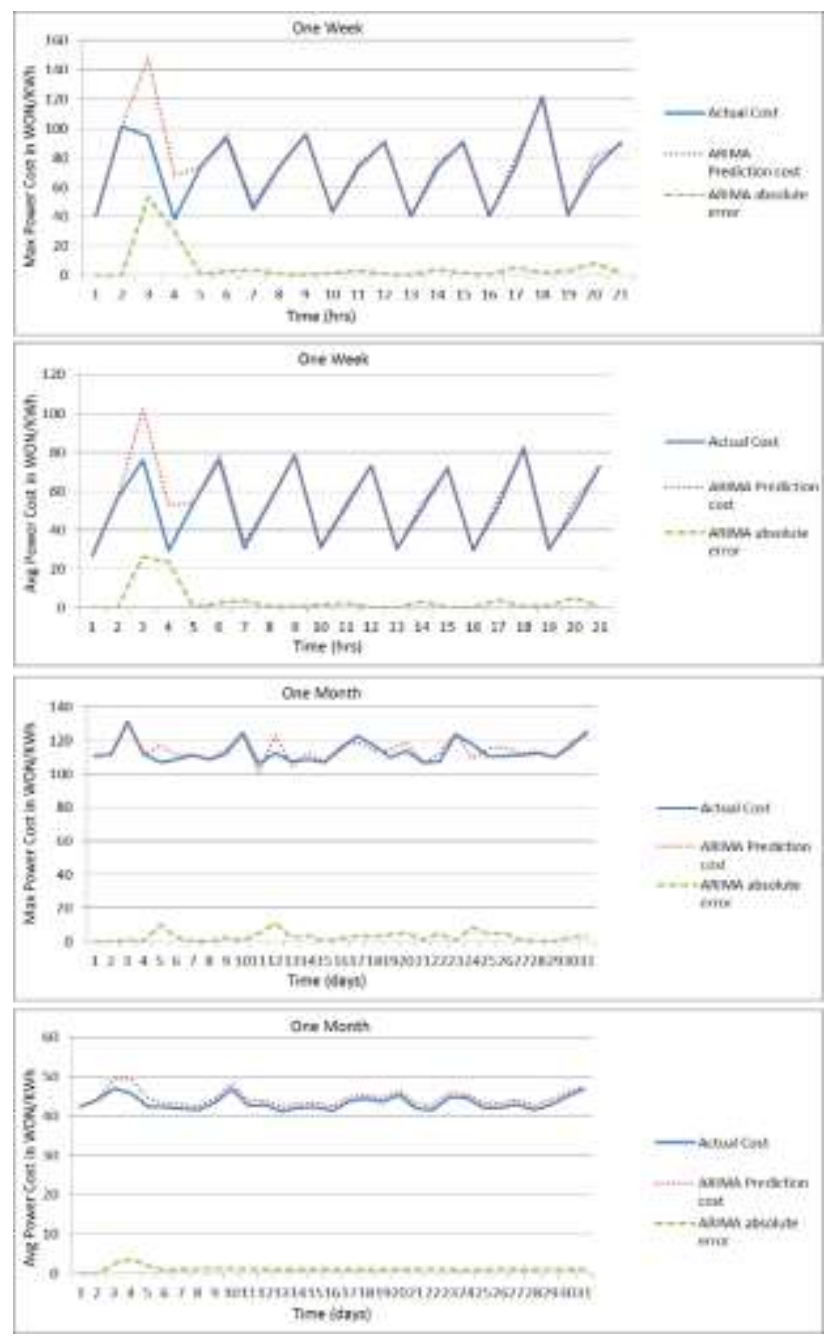

Fig. 5. Actual Cost, Predicted Cost using ARIMA $(0,1,1)$ Model and Absolute Error based on the Power Consumption Scenarios.
The aim of testing ARIMA model on different power consumption scenarios is to authenticate the efficiency of the prediction algorithm. In order to estimate daily electricity cost for each day of the week and month, the general electricity price and power consumption data are collected for one month of January 2010. From the Fig. 5 we can see that (AE) for weekly basis electricity cost prediction is very less except for the hour $4 \& 5$. The performance of the ARIMA model for the maximum and average weekly electricity cost prediction is good.

Fig. 5 also shows the efficiency of the proposed ARIMA $(0,1,1)$ model based on the monthly power consumption scenario. The (AE) values in Fig. 5 also describe the efficiency of the proposed algorithm for prediction of electricity cost on monthly basis. The maximum and average monthly electricity predicted cost is very good by looking into (AE) values. The $\mathrm{AE}$ values are much near to zero which means the error between actual cost and predicted cost is less and almost zero.

So the bottom line is that proposed ARIMA $(0,1,1)$ model effectively predicted the electricity cost using different power consumption scenarios.

\section{CONCLUSIONS}

The management of energy system depends on the good knowledge of the electricity cost due to the deregulation of the energy market prices and cost. In the literature a number of different simulation and prediction methodologies have been proposed and applied for a number of times to forecast the electricity prices. These methodologies can also be used for power cost prediction. The Autoregressive Integrated Moving Average (ARIMA) method is an optimal prediction technique and has been established and used successfully previously in other fields for prediction. In this paper we proposed it for electricity cost prediction. The prediction results of the ARIMA $(0,1,1)$ model is satisfactory. The efficiency and reliability of the ARIMA $(0,1,1)$ method is verified using a case study in Republic of Korea. The proposed methodology has many benefits with respect to electricity cost. The proposed technique validates the effectiveness of ARIMA ( 0 , $1,1)$ approach for electricity cost prediction.

Electricity cost of Republic of Korea reveal periodicity and time-varying uncertainty credited to the non-storability of electricity, insignificant costs and potential for electricity power market. Our aim is to propose and evaluate a technique to separate periodicity that could be effortlessly reasonable in application. The proposed $\operatorname{ARIMA}(0,1,1)$ technique also produced good results which are reasonably acceptable for current research. The proposed ARIMA $(0,1,1)$ technique takes into account the periodicity of the electricity cost data and up-to somehow can successfully solve the prediction issue along with periodicity. The proposed model is essentially simple, typical and do not need to make tough variations and judgments concerning the clear form of the model for different trends of the power cost.

The proposed prediction technique using $\operatorname{ARIMA}(0,1,1)$ model results in minimum AE. Finally based on the results and above arguments we can say that the ARIMA $(0,1,1)$ 
prediction technique performs satisfactory up-to some level. From the above discussions, it is vibrant that the proposed ARIMA $(0,1,1)$ model is effective for electricity cost prediction.

There is limitation of ARIMA model to predict electricity cost and price. If there is various kind of non-linearity in the data and there is no specific patterns in the data, then $\mathrm{AE}$ values getting increases which affects the accuracy rate.

\section{ACKNOWLEDGMENT}

This research is supported by Energy Cloud R\&D Program via the National Research Foundation of Korea (NRF) funded by the Ministry of Science, ICT (2019M3F2A1073387), and this research is also supported by Institute for Information \& communications Technology Planning \& Evaluation (IITP) grant funded by the Korea government (MSIT)(No.2018-001456, AutoMaTa: Autonomous Management framework based on artificial intelligent Technology for adaptive and disposable IoT). Any correspondence related to this paper should be addressed to Dohyeun Kim.

\section{REFERENCES}

[1] H.Y. Yamin; S.M. Shahidehpour, and Z. Li, "Adaptive short-term electricity price forecasting using artificial neural networks in the restructured power markets," Electr Power Energy Syst, vol. 26, pp. 571-81, 2004.

[2] P. Mandal, T. Senjyu, N. Urasaki, T. Funabshi, and A. K. Srivastava, “A novel approach to forecast electricity price for PJM using neural network and similar days method," IEEE Trans Power Syst, vol. 22, pp. 2058-2065, 2007.

[3] H. T. Pao, "Forecasting electricity market pricing using artificial neural networks," Energy Convers Manage, vol. 48, pp. 907-912, 2007.

[4] R. Pino, J. Parreno, A. Gomez, and P. Priore, "Forecasting next-day price of electricity in the Spanish energy market using artificial neural networks," Eng Appl Artif Intell, vol. 21, pp. 53-62, 2008.

[5] N. M. Pindoriya, S. N. Singh, and S. K. Singh, "An adaptive wavelet neural network-based energy price forecasting in electricity markets," IEEE Trans Power Syst, vol. 23, pp. 1423-1432, 2008.

[6] N. Amjady, A. Daraeepour, and F. Keynia, "Day-ahead electricity price forecasting by modified relief algorithm and hybrid neural network," IET Generat Trans Distribut, vol. 4, pp. 432-444, 2010.

[7] G. Raquel, M. Romeo-Luis, and A. Gil, "Forecasting of electricity prices with neural networks," Int J Energy Convers Manage, vol. 47, pp. 1770$1778,2006$.
[8] J. P. S. Catalao, S. J. P. S. Mariano, V. M. F. Mendes, and L. A. F. M. Ferreira, "Short-term electricity prices forecasting in a competitive market: a neural network approach," Electr Power Syst Res, vol. 77, pp. 1297-1304, 2007.

[9] R. Lapedes, R. Farber, "Nonlinear signal processing using neural networks: prediction and system modeling," Technical report LA-VR872662. Los Alamos, New Mexico: Los Alamos National Laboratory, 1987.

[10] E. R. J. Van, "The application of neural network in the forecasting of share prices," Finance and Technology Publishing, 1996.

[11] Y. Y. Hong, and C. F. Lee, "A neuro-fuzzy price forecasting approach in deregulated electricity markets," Elect Power Syst Res, vol. 73, pp. 151$157,2005$.

[12] C. P. Rodriguez, and G. J. Anders, "Energy price forecasting in the Ontario competitive power system market," IEEE Trans Power Syst, vol. 19, pp. 366-374, 2004.

[13] G. Li, C. C. Liu, C. Mattson, and J. Lawarree, "Day-ahead electricity price forecasting in a grid environment," IEEE Trans Power Syst, vol. 22, pp. 266-274, 2007.

[14] F. J. Nogales, J. Contreras, A. J. Conejo, and R. Espinola, "Forecasting next-day electricity prices by time series models," IEEE Trans Power Syst, vol. 17, pp. 342-348, 2002.

[15] M. Shafie-Khah, M. M. Parsa, and Sheikh-El-Eslami, "Price forecasting of day-ahead electricity markets using a hybrid forecast method," Energy Conversion and Management vol. 5, pp. 2165-2169, 2011.

[16] Lin. Whei-Min, Gow. Hong-Jey, Tsai. Ming-Tang, "Electricity price forecasting using Enhanced Probability Neural Network," Energy and Conversion Management vol. 51, pp. 2707-2714, 2010.

[17] H. Shayeghi, and A. Ghasemi, "Day-ahead electricity prices forecasting by a modified CGSA technique and hybrid WT in LSSVM based scheme," Energy Conversion and Management vol. 74, pp. 482-491, 2013.

[18] Z. Yuan, W. Wang, H. Wang, N. Razmjooy, "A new technique for optimal estimation of the circuit-based PEMFCs using developed Sunflower Optimization Algorithm," Energy Rep, vol. 6, pp. 662-671. 2020.

[19] Z. Yang, Q. Liu, L. Zhang, J. Dai, N. Razmjooy, "Model parameter estimation of the PEMFCs using improved Barnacles Mating Optimization algorithm," Energy Rep, vol. 212, pp. 0360-5442, 2020.

[20] Y. Guo, X. Dai, K. Jermsittiparsert, N. Razmjooy, “An optimal configuration for a battery and PEM fuel cell-based hybrid energy system using developed Krill herd optimization algorithm for locomotive application," Energy Rep, vol. 6, pp. 885-894, 2020.

[21] X. Fan, H. Sun, Z. Yuan, Z. Li, R. Shi, N. Razmjooy, "Multi-objective optimization for the proper selection of the best heat pump technology in a fuel cell-heat pump micro-CHP system," Energy Rep, vol. 6, pp. 325$335,2020$. 